\title{
AN ABELIAN GROUP ASSOCIATED WITH TOPOLOGICAL DYNAMICS
}

\author{
KAZUHIRO KAWAMURA
}

\begin{abstract}
For a continuous surjection $T: X \rightarrow X$ on a compact metric space $X$ and a unimodular continuous weight on $X$, we consider a weighted composition operator $U_{T, w}$ on the Banach space $C(X)$ of complex-valued continuous functions on $X$ with the sup norm. The set $\mathcal{W}_{T}$ of all weights $w$, for which the operator $U_{T, w}$ has an eigenvalue with a unimodular eigenfunction, forms a topological abelian group. The group $\mathcal{W}_{T}$ admits a homomorphism $W_{T}$ to the first integral Cech cohomology of the space $X$. The image and the kernel of $W_{T}$ carry topological and ergodic aspects of the dynamics $T$. A concrete description of $\operatorname{Im} W_{T}$ and $\operatorname{Ker} W_{T}$ is given for positively expansive eventually-onto open maps (under an assumption on the induced homomorphism of the first Čech cohomology) and minimal rotations on tori.
\end{abstract}

1. Introduction. For a compact metric space $X, C(X)$ denotes the Banach space of all complex-valued continuous functions on $X$ with the sup norm. For a continuous surjection $T: X \rightarrow X$ (referred to as a discrete continuous dynamical system) and a continuous function $w$ : $X \rightarrow \mathbb{C}$ (referred to as a weight function), a bounded linear operator, called a weighted composition operator, $U_{T, w}: C(X) \rightarrow C(X)$, is defined by the following formula:

$$
\left(U_{T, w} f\right)(x)=w(x) \cdot f(T(x)) \quad(f \in C(X) \text { and } x \in X) .
$$

Throughout the present paper, we assume that $w$ is a unimodular function, that is, a continuous function such that $|w(x)|=1$ for each

2010 AMS Mathematics subject classification. Primary 37B05, Secondary $46 \mathrm{E} 15$.

Keywords and phrases. Topological transitivity, weighted composition operator, Banach space of continuous functions.

This research is partially supported by JSPS Grant-In-Aid No. 22540062.

Received by the editors on October 19, 2011, and in revised form on September 9, 2013. 
$x \in X$. This assumption, together with the surjectivity of $T$, make $U_{T, w}: C(X) \rightarrow C(X)$ an isometric linear operator.

The operator $U_{T, w}$ may or may not have an eigenvalue. We consider the set $\mathcal{W}_{T}$ of all weights $w$, for which $U_{T, w}$ has an eigenvalue with a unimodular eigenfunction. The set $\mathcal{W}_{T}$ forms a topological abelian group under pointwise multiplication, and it admits a continuous homomorphism $W_{T}: \mathcal{W}_{T} \rightarrow \check{\mathrm{H}}^{1}(X ; \mathbb{Z})$ to the integral first Čech cohomology of $X$ with the discrete topology (see Section 2, Definition 2.1). The image $\operatorname{Im} W_{T}$ is described by the homomorphisms induced by $T$ (see Theorem 2.2), while the kernel $\operatorname{Ker} T$ is related to the space of real continuous coboundaries of $T$ (see Theorem 2.8 and Theorem 2.9). In this sense, $\mathcal{W}_{T}$ carries topological and ergodic aspects of the dynamics, $T$. While the framework presented here is applied to arbitrary continuous surjections, special attention will be paid to positively expansive open maps which are eventually onto and minimal rotations on tori, dynamical systems that have been studied by many authors. A concrete description of $\operatorname{Im} W_{T}$ and $\operatorname{Ker} W_{T}$ is available for such a $T$ (under an assumption on the induced homomorphism on the first Čech cohomology), see Corollary 2.11.

The study of composition operators has a long history in ergodic theory $([\mathbf{1 2}, \mathbf{2 1}]$, etc.). The set up of the present paper is, of course, very close to the one for the operators on $L^{\infty}$ spaces of suitable probability measures. Still, the author hopes that the present approach sheds light on the study of topological dynamics.

In the rest of this section, we fix notation, give some definitions and recall some auxiliary results. Throughout, $\mathbb{T}$ denotes the unit circle of the complex plane $\mathbb{C}: \mathbb{T}=\{z \in \mathbb{C}|| z \mid=1\}$. The $n$-dimensional torus is denoted by $\mathbb{T}^{n}$. For compact metric space $X, C(X)$ (respectively, $\left.C_{\mathbb{R}}(X)\right)$ denotes the Banach space of all complex-valued (respectively, real-valued) continuous functions on $X$ with the sup norm $\|\cdot\|_{\infty}$. For two compact metric spaces $X$ and $Y, \operatorname{Map}(X, Y)$ denotes the space of all continuous maps of $X$ to $Y$ with the sup metric. In particular, $C(X)=\operatorname{Map}(X, \mathbb{C})$ and $C_{\mathbb{R}}(X)=\operatorname{Map}(X, \mathbb{R})$.

The Čech cohomology with integer coefficient is denoted by $\check{\mathrm{H}}^{*}(X ; \mathbb{Z})$. We only deal with the first cohomology and make use of the natural isomorphism

$$
\check{\mathrm{H}}^{1}(X ; \mathbb{Z}) \cong[X, \mathbb{T}]
$$


where $[X, \mathbb{T}]$ denotes the group of free homotopy classes of the maps of $X$ to $\mathbb{T}$ with pointwise multiplication as the additive operation. When $G$ is a compact topological group, there exists a canonical isomorphism $\check{\mathrm{H}}^{1}(G ; \mathbb{Z}) \cong G^{\wedge}$ between the first Cech cohomology of $G$ and the character group $G^{\wedge}$ so that, for each continuous homomorphism $f: G \rightarrow G$, the induced homomorphism $f^{*}: \check{\mathrm{H}}^{1}(G ; \mathbb{Z}) \rightarrow \check{\mathrm{H}}^{1}(G ; \mathbb{Z})$ is identified with the homomorphism $f^{\wedge}: G^{\wedge} \rightarrow G^{\wedge}$ defined by $f^{\wedge}(\chi)=\chi \circ f, \chi \in G^{\wedge}$.

A continuous map $T: X \rightarrow T$ of a compact metric space $(X, d)$ is said to be positively expansive if there exists a constant $c>0$ such that, for each pair $x, y$ of distinct points of $X$, there exists a non-negative integer $n$ such that $d\left(T^{n}(x), T^{n}(y)\right)>c$. The positive expansiveness is a topological notion in that it does not depend on the choice of the metric $d$. It is known that the positively expansive open map $T: X \rightarrow X$ is a local homeomorphism: each point $x$ of $X$ has an open neighborhood $U$ such that $T \mid U: U \rightarrow T(U)$ is a homeomorphism. For more details, see $[\mathbf{1}, \mathbf{3}, \mathbf{8}, \mathbf{1 6}, \mathbf{1 7}, \mathbf{1 8}]$, etc. A minimal map $T: X \rightarrow X$ is a continuous map on $X$ such that the orbit $\left\{T^{n}(x) \mid n \geq 0\right\}$ of $x$ is dense for each $x \in X$ (see $[\mathbf{9}, \mathbf{2 1}]$, etc.). Also, we say that a map $T$ is eventually onto if each nonempty open set $U$ admits a non-negative integer $k$ such that $T^{k}(U)=X$.

The references $[\mathbf{2}, \mathbf{2 1}]$ are excellent expositions on the basics of topological dynamics and ergodic theory.

2. The group $\mathcal{W}_{T}$. Let us start with a preliminary consideration. Suppose, for a moment, that $T: X \rightarrow X$ is topologically transitive, that is, there exists a point $x_{0} \in X$ such that the orbit $\left\{T^{n}\left(x_{0}\right) \mid n \geq 0\right\}$ is dense in $X$. For a unimodular weight $w$, suppose that $U_{T, w}$ has an eigenvalue $\lambda$ with an eigenfunction $f \in C(X)$. Since $U_{T, w}: C(X) \rightarrow$ $C(X)$ is an isometry, we have $|\lambda|=1$. By definition, we have

$$
w(x) \cdot f(T(x))=\lambda \cdot f(x)
$$

for each $x \in X$. Taking the moduli of both sides of (2.1), we see $|f(T(x))|=|f(x)|$. In particular, we have $\left|f\left(T^{n}\left(x_{0}\right)\right)\right|=\left|f\left(x_{0}\right)\right|$ for each non-negative integer $n$. Because of the denseness of the orbit $\left\{T^{n}\left(x_{0}\right) \mid n \geq 0\right\}$ and the continuity of $f$, we see that the function $|f|$ is a constant function: $|f| \equiv\left|f\left(x_{0}\right)\right|$. Dividing both sides of (2.1) by 
$\left|f\left(x_{0}\right)\right|$, we see that the map $\bar{f}:=f /\left|f\left(x_{0}\right)\right| \in \operatorname{Map}(X, \mathbb{T})$ can be chosen as an eigenfunction of $\lambda$.

The above observation leads us to the following definition.

Definition 2.1. Let $T: X \rightarrow X$ be a continuous surjection on a compact metric space $X$. Let $\mathcal{W}_{T}$ be the topological abelian group defined by

$$
\begin{aligned}
& \mathcal{W}_{T}=\{w \in \operatorname{Map}(X, \mathbb{T}) \mid \\
& \text { there exist } \left.\lambda \in \mathbb{T} \text { and } f \in \operatorname{Map}(X, \mathbb{T}) \text { such that } U_{T, w} f=\lambda \cdot f\right\},
\end{aligned}
$$

with pointwise multiplication and compact-open topology.

The group $\mathcal{W}_{T}$ admits a natural homomorphism $W_{T}: \mathcal{W}_{T} \rightarrow$ $\check{\mathrm{H}}^{1}(X ; \mathbb{Z})$ as follows. For $w \in \mathcal{W}_{T}$, let $[w]$ be the homotopy class in $[X, \mathbb{T}]$ represented by $w$. Recalling the isomorphism $\check{\mathrm{H}}^{1}(X ; \mathbb{Z}) \cong[X, \mathbb{T}]$, the map defined by

$$
W_{T}(w)=[w] \in[X, \mathbb{T}] \cong \check{\mathrm{H}}^{1}(X ; \mathbb{Z}), \quad w \in \mathcal{W}_{T},
$$

is a homomorphism. With $\check{\mathrm{H}}^{1}(X ; \mathbb{Z})$ being endowed with the discrete topology, we see easily that $W_{T}$ is a continuous homomorphism.

First, we examine the image $\operatorname{Im} W_{T} \subset \check{\mathrm{H}}^{1}(X ; \mathbb{Z})$.

Theorem 2.2. Let $T: X \rightarrow X$ be a continuous surjection with the induced homomorphism $T^{*}: \check{\mathrm{H}}^{1}(X ; \mathbb{Z}) \rightarrow \check{\mathrm{H}}^{1}(X ; \mathbb{Z})$. Then we have $\operatorname{Im} W_{T}=\operatorname{Im}\left(T^{*}-\mathrm{id}\right)$.

Proof. For each $w \in \mathcal{W}_{T}$, we take $\lambda \in \mathbb{T}$ and $f \in \operatorname{Map}(X, \mathbb{T})$ such that $w \cdot(f \circ T)=\lambda \cdot f($ see $(1.1))$. Noticing that the multiplication map $m_{\lambda}$ by $\lambda$ :

$$
m_{\lambda}: \mathbb{T} \longrightarrow \mathbb{T}, \quad m_{\lambda}(z)=\lambda z, \quad z \in \mathbb{T},
$$

is homotopic to $\mathrm{id}_{\mathbb{T}}$, we obtain the following equality in $\check{\mathrm{H}}^{1}(X ; \mathbb{Z})$ :

$$
[w]+T^{*}([f])=[f]
$$

which implies $[w] \in \operatorname{Im}\left(T^{*}-\mathrm{id}\right)$. 
Conversely, let $w \in \operatorname{Map}(X, \mathbb{T})$ be a map such that $[w]=\left(T^{*}-\mathrm{id}\right)[g]$ for some $[g] \in \check{\mathrm{H}}^{1}(X ; \mathbb{Z})$. In terms of homotopy, this means

$$
w \simeq(g \circ T) \cdot g^{-1} .
$$

Hence, there exists a function $\varphi \in C_{\mathbb{R}}(X)$ such that $w \cdot\left(g^{-1} \circ T\right) \cdot g=$ $\exp (i \varphi)$. Let $f=g^{-1}$. Then we have

$$
(w \cdot \exp (-i \varphi)) \cdot(f \circ T)=f .
$$

Hence, $w \cdot \exp (-i \varphi) \in \mathcal{W}_{T}$. For $0 \leq t \leq 1$, let $w_{t}=w \cdot \exp (-i t \varphi)$. Then $\left(w_{t}\right)_{0 \leq t \leq 1}$ is a homotopy between $w$ and $w \cdot \exp (-i \varphi)$. Thus, we see $[w]=[w \cdot \exp (-i \varphi)] \in \operatorname{Im} W_{T}$.

This completes the proof.

\section{Example 2.3.}

(1) For each integer $n \geq 2$, let $p_{n}: \mathbb{T} \rightarrow \mathbb{T}$ be the map defined by $p_{n}(z)=z^{n}, z \in \mathbb{T}$. The map $p_{n}$ is positively expansive, open and eventually onto. By Theorem 2.2 , we have $\operatorname{Im} W_{p_{n}} \cong(n-1) \mathbb{Z} \subset \mathbb{Z}$.

For example, the weight function $w: \mathbb{T} \rightarrow \mathbb{C}$ defined by $w(z)=z$ belongs to $\mathcal{W}_{p_{2}}$ but does not belong to $\mathcal{W}_{p_{n}}$ for each $n \geq 3$.

(2) Let $\Sigma_{n}$ be the $n$-adic solenoid. This is the inverse limit space defined by

$$
\Sigma_{n}=\lim _{\leftarrow}\left(\mathbb{T} \longleftarrow p_{n} \mathbb{T} \longleftarrow p_{n} \mathbb{T} \longleftarrow p_{n} \cdots\right)
$$

with all bonding maps being equal to $p_{n}$ of (1) above. For each integer $m>n, \widehat{p}_{m}: \Sigma_{n} \rightarrow \Sigma_{n}$ defined by

$$
\widehat{p}_{m}\left(z_{1}, z_{2}, \ldots\right)=\left(z_{1}^{m}, z_{2}^{m}, \ldots\right)
$$

is a positively expansive, open and eventually onto map. It is known that $\check{\mathrm{H}}^{1}\left(\Sigma_{n} ; \mathbb{Z}\right) \cong\left\{\frac{k}{n^{i}} \mid i, k \in \mathbb{Z}, i \geq 0\right\}$. Applying Theorem 2.2, we have

$$
\operatorname{Im} W_{\hat{p}_{m}}=(m-1) \check{\mathrm{H}}^{1}\left(\Sigma_{n} ; \mathbb{Z}\right) \cong\left\{\frac{(m-1) k}{n^{i}} \mid i, k \in \mathbb{Z}, i \geq 0\right\} .
$$

(3) For a positive integer $n \geq 1$, let $A$ be an $n \times n$ integral matrix such that each eigenvalue has modulus $>1$. Then $A$ induces a positively expansive open map $T_{A}: \mathbb{T}^{n} \rightarrow \mathbb{T}^{n}$ of $n$-dimensional torus $\mathbb{T}^{n}$ which is eventually onto. For $n \geq 2$, this is a higher dimensional analogue 
of (1). Noticing that

$$
\check{\mathrm{H}}^{1}\left(\mathbb{T}^{n} ; \mathbb{Z}\right) \cong \mathbb{Z}^{n} \text { and } T_{A}^{*}=A: \check{\mathrm{H}}^{1}\left(\mathbb{T}^{n} ; \mathbb{Z}\right) \rightarrow \check{\mathrm{H}}^{1}\left(\mathbb{T}^{n} ; \mathbb{Z}\right),
$$

we obtain, by Theorem $2.2, \operatorname{Im} W_{T_{A}}=\operatorname{Im}\left(A-\mathrm{id}_{\mathbb{Z}^{n}}\right)$.

(4) Let $G$ be a compact abelian metric group, and let $T: G \rightarrow G$ be a positively expansive open homomorphism on $G$ which is eventually onto. When $G$ is a Lie group, such a map is topologically conjugate to the map $T_{A}$ of (3) [19].

For a non-Lie-group example, see [1] in which positively expansive open maps on solenoidal groups are characterized. Under the identification of $\check{\mathrm{H}}^{1}(G ; \mathbb{Z})$ with the character group $G^{\wedge}$, we see

$$
\operatorname{Im} W_{T}=\left\{(\chi \circ T) \cdot \chi^{-1} \mid \chi \in G^{\wedge}\right\} .
$$

(5) Let $\mu^{n}$ be the universal Menger compactum [5] (cf., [7, 13]). By [20, Corollary 2.3] (and its proof), $\mu^{n}$ admits a positively expansive open map $T: \mu^{n} \rightarrow \mu^{n}$ which is eventually onto. The first cohomology is given as follows:

$$
\check{\mathrm{H}}^{1}\left(\mu^{n} ; \mathbb{Z}\right) \cong\left\{\begin{array}{rl}
\oplus_{\infty} \mathbb{Z} & n=1 \\
0 & n \geq 2 .
\end{array}\right.
$$

In particular, we see $\operatorname{Im} W_{T}=0$ for each $n \geq 2$.

Proposition 2.4. Let $G$ be a compact connected abelian group. For an element $\alpha$ of $G$, let $r_{\alpha}: G \rightarrow G$ be the rotation defined by $r_{\alpha}(x)=\alpha x, x \in G$. Then $\operatorname{Im} W_{r_{\alpha}}=0$.

Proof. Every compact connected abelian metric group is the limit of an inverse sequence of (finite dimensional) tori [11, Remark 2.35]. So, let $G=\lim _{\leftarrow}\left(G_{n}, p_{n}: G_{n+1} \rightarrow G_{n}\right)$ where each $G_{n}$ is a torus and $p_{n}$ is a continuous epimorphism. Let $\pi_{n}: G \rightarrow G_{n}$ be the projection which is a homomorphism, and let $\alpha_{n}=\pi_{n}(\alpha) \in G_{n}$. It is easy to see that $r_{\alpha}=\lim _{\leftarrow} r_{\alpha_{n}}$. Since each $r_{\alpha_{n}}: G_{n} \rightarrow G_{n}$ is homotopic to $\operatorname{id}_{G_{n}}$, we have $r_{\alpha_{n}}^{*}=\mathrm{id}_{G_{n}}$. Therefore, $r_{\alpha}^{*}=\lim _{\rightarrow} r_{\alpha_{n}}^{*}=\operatorname{id}_{\check{\mathrm{H}}^{1}(G ; \mathbb{Z})}$. Theorem 2.2 implies the desired conclusion.

In order to examine the kernel of $W_{T}$, we introduce the following.

Definition 2.5. Let $T: X \rightarrow X$ be a continuous map of a compact metric space $X$. A real-valued continuous function $f \in C_{\mathbb{R}}(X)$ is called 
a real continuous coboundary with respect to $T$ if there exists a function $g \in C_{\mathbb{R}}(X)$ such that $f=g \circ T-g$. The space of all continuous coboundaries is denoted by $\operatorname{Cob}_{\mathbb{R}}(T)$.

Let $C_{0}(X ; \mathbb{R})$ be the closed subspace of $C_{\mathbb{R}}(X)$ defined by

$$
C_{0}(X ; \mathbb{R})=\left\{f \in C_{\mathbb{R}}(X) \mid \int_{X} f d m=0\right.
$$

for each $T$-invariant probability measure $m$ on $X$ \}.

It is well known that $\operatorname{Cob}_{\mathbb{R}}(T)$ is a dense subspace of $C_{0}(X ; \mathbb{R})$. For a proof, see [14, Lemma 1]. While in [14, Lemma 1], the surjection $T$ is assumed to be a homeomorphism, its proof can easily be modified to a proof for general surjections.

The abelian group $\mathbb{T}$ is regarded as a subgroup of $\operatorname{Map}(X, \mathbb{T})$ by the identification:

$$
z \longmapsto \text { the constant function } \equiv z \text {. }
$$

Also, we consider the following $\operatorname{subgroup} \exp \left(i \operatorname{Cob}_{\mathbb{R}}(T)\right)$ of $\operatorname{Map}(X, \mathbb{T})$ :

$$
\exp \left(i \operatorname{Cob}_{\mathbb{R}}(T)\right):=\left\{\exp (i \varphi) \mid \varphi \in \operatorname{Cob}_{\mathbb{R}}(T)\right\} .
$$

Lemma 2.6. Assume that $X$ is connected. Then we have $\mathbb{T} \cap$ $\exp \left(i \operatorname{Cob}_{\mathbb{R}}(T)\right)=\{1\}$.

Proof. Take a function $f \in \mathbb{T} \cap \exp \left(i \operatorname{Cob}_{\mathbb{R}}(T)\right)$, and let $f=\exp (i(\varphi \circ$ $T-\varphi))=\exp (i \theta)$ for some $\varphi \in C_{\mathbb{R}}(X)$ and $\theta \in \mathbb{R}$. There exists a function $\nu: X \rightarrow \mathbb{Z}$ such that $\varphi \circ T-\varphi=\theta+2 \pi \nu$. From this, we see that the function $\nu$ is continuous and, by the connectedness of $X$, is a constant function. By induction, we obtain

$$
\varphi \circ T^{n}-\varphi=n(\theta+2 \pi \nu)
$$

for each positive integer $n$. Then

$$
|n(\theta+2 \pi \nu)| \leq\left\|\varphi \circ T^{n}\right\|_{\infty}+\|\varphi\|_{\infty}=2\|\varphi\|_{\infty},
$$

for each $n$. Hence, $\theta=-2 \pi \nu$, and hence $\varphi \circ T=\varphi$, which implies $f \equiv 1$.

This completes the proof. 
Remark 2.7. The above lemma does not hold in general when $X$ is disconnected.

An easy example that illustrates the above remark is given below.

Let $X=\{1,2,3\}$ be the three points set with the discrete topology and let $T: X \rightarrow X$ be the map defined by $T(i)=i+1(\bmod 3)$. For the function $g(i)=i / 3, i=1,2,3$, define a coboundary $\varphi$ by $\varphi=2 \pi(g \circ T-g)$. Then we have

$$
\exp (i \varphi) \equiv \exp (i(2 \pi / 3)) \neq 1
$$

hence, $\exp \left(i\left(\operatorname{Cob}_{\mathbb{R}}(T)\right)\right) \cap \mathbb{T} \neq\{1\}$.

By making use of Lemma 2.6, the kernel of $W_{T}$ of some dynamics $T$ is described as follows:

Theorem 2.8. Let $T: X \rightarrow X$ be a continuous surjection on a compact metric space $X$.

(i) Assume that the homomorphism $T^{*}-\mathrm{id}_{\check{\mathrm{H}}^{1}(X ; \mathbb{Z})}: \check{\mathrm{H}}^{1}(X ; \mathbb{Z}) \rightarrow$ $\check{\mathrm{H}}^{1}(X ; \mathbb{Z})$ is injective. Then we have

$$
\operatorname{Ker} W_{T}=\mathbb{T} \cdot \exp \left(i \operatorname{Cob}_{\mathbb{R}}(T)\right) .
$$

In particular, if $\check{\mathrm{H}}^{1}(X ; \mathbb{Z})=0$, then the above equality holds.

(ii) Suppose moreover, that $X$ is connected. Then we have an isomorphism

$$
\operatorname{Ker} W_{T} \cong \mathbb{T} \oplus \exp \left(i \operatorname{Cob}_{\mathbb{R}}(T)\right)
$$

Proof. In order to prove (i), first let $\varphi$ be a real continuous coboundary of $T$ and $\omega \in \mathbb{T}$. Take a function $\alpha \in C_{\mathbb{R}}(X)$ such that $\varphi=\alpha \circ T-\alpha$, and define $f:=\exp (-i \alpha)$. Then, for each $x \in X$, we have

$$
\begin{aligned}
\omega \cdot \exp (i \varphi) \cdot(f \circ T) & =\omega \cdot \exp (i \varphi) \cdot \exp (-i(\alpha \circ T)) \\
& =\omega \cdot \exp (-i \alpha)=\omega \cdot f .
\end{aligned}
$$

Hence, $w:=\omega \cdot \exp (i \varphi)$ is an element of $\mathcal{W}_{T}$ such that $w \simeq \exp (i \varphi) \simeq 0$. Thus, we see $w \in \operatorname{Ker} W_{T}$.

Conversely, take an arbitrary $w \in \operatorname{Ker} W_{T}$. Then $w \simeq 0$, and there exists a function $\varphi \in C_{\mathbb{R}}(X)$ such that $w=\exp (i \varphi)$. Let $\lambda=\exp (i \theta)$ 
be an eigenvalue of $U_{T, w}$ with an eigenfunction $f \in \operatorname{Map}(X, \mathbb{T})$. The equation $w \cdot(f \circ T)=\lambda \cdot f$ is re-written as

$$
\exp [i(\varphi(x)-\theta)] \cdot f(T(x))=f(x) \quad(x \in X) .
$$

Let $\eta(x)=\varphi(x)-\theta$. Noticing $[\exp (i \eta)]=0 \in \check{\mathrm{H}}^{1}(X ; \mathbb{Z})$, we obtain the following equality in $\check{\mathrm{H}}^{1}(X ; \mathbb{Z})$ :

$$
T^{*}([f])=[\exp (i \eta)]+T^{*}([f])=[f] .
$$

Since $T^{*}-$ id is injective, we obtain $[f]=0 \in \check{\mathrm{H}}^{1}(X ; \mathbb{Z})$, and thus $f \simeq 0: X \rightarrow \mathbb{T}$. Hence, there exists a function $\alpha \in C_{\mathbb{R}}(X)$ such that $f=\exp (i \alpha)$. Substituting this into equation (2.3), we obtain an integer-valued function $\nu: X \rightarrow \mathbb{Z}$ such that

$$
\eta(x)+\alpha(T(x))-\alpha(x)=2 \pi \nu(x)
$$

for each $x \in X$. Let $\psi=-(\alpha \circ T-\alpha)$. The function $\psi$ is a coboundary of $T$. Now we see that the function $w$ has the desired form as follows:

$$
\begin{aligned}
w=\exp (i \varphi) & =\exp (i \theta) \exp (i \eta) \\
& =\exp (i \theta) \exp (i \psi) \exp (2 \pi i \nu) \\
& =\exp (i \theta) \exp (i \psi)=\lambda \exp (i \psi) .
\end{aligned}
$$

Therefore, we obtain the equality $\operatorname{Ker} W_{T}=\mathbb{T} \cdot \exp \left(i \operatorname{Cob}_{\mathbb{R}}(T)\right)$, as desired.

(ii) is a direct consequence of (i) and Lemma 2.6. This completes the proof.

Theorem 2.8 applies to the dynamics of Example 2.3. Rotations on tori (Proposition 2.4) require a separate argument. Recall from Proposition 2.4 that $\mathcal{W}_{r_{\alpha}}=\operatorname{Ker} W_{r_{\alpha}}$. An explicit description of the group structure of $\mathcal{W}_{r_{\alpha}}$ for a general compact abelian metric group is not known to the author.

Theorem 2.9. Let $r_{\alpha}: \mathbb{T}^{n} \rightarrow \mathbb{T}^{n}$ be the rotation on the $n$-dimensional torus by an element $\alpha \in \mathbb{T}^{n}$. Then we have an isomorphism

$$
\mathcal{W}_{r_{\alpha}} \cong \mathbb{T} \oplus \exp \left(i \operatorname{Cob}_{\mathbb{R}}\left(r_{\alpha}\right)\right)
$$

Proof. Let $\alpha=\left(\alpha_{1}, \ldots, \alpha_{n}\right) \in \mathbb{T}^{n}$ with $\alpha_{i} \in \mathbb{T}, i=1, \ldots, n$. In view of Lemma 2.6, it suffices to prove that $\mathcal{W}_{r_{\alpha}}=\mathbb{T} \cdot \exp \left(i \operatorname{Cob}_{\mathbb{R}}\left(r_{\alpha}\right)\right)$. 
The inclusion $\mathcal{W}_{r_{\alpha}} \supset \mathbb{T} \cdot \exp \left(i \operatorname{Cob}_{\mathbb{R}}\left(r_{\alpha}\right)\right)$ is proved in exactly the same way as the one of (2.2) in Theorem 2.8. So the reverse inclusion

$$
\mathcal{W}_{r_{\alpha}} \subset \mathbb{T} \cdot \exp \left(i \operatorname{Cob}_{\mathbb{R}}\left(r_{\alpha}\right)\right)
$$

remains to be shown. Let $w \in \mathcal{W}_{r_{\alpha}}$, and take $\lambda \in \mathbb{T}$ and $f \in$ $\operatorname{Map}\left(\mathbb{T}^{n}, \mathbb{T}\right)$ such that

$$
w \cdot\left(f \circ r_{\alpha}\right)=\lambda \cdot f .
$$

The induced homomorphism $f_{\sharp}: \pi_{1}\left(\mathbb{T}^{n}\right) \cong \mathbb{Z}^{n} \rightarrow \pi_{1}(\mathbb{T}) \cong \mathbb{Z}$ has the form:

$$
f_{\sharp}\left(k_{1}, \ldots, k_{n}\right)=\sum_{i=1}^{n} \mu_{i} k_{i}, \quad\left(k_{1}, \ldots, k_{n}\right) \in \mathbb{Z}^{n}
$$

for some $\mu=\left(\mu_{1}, \ldots, \mu_{n}\right)$. Let $p_{\mu}: \mathbb{T}^{n} \rightarrow \mathbb{T}$ be the map defined by

$$
p_{\mu}(z)=\prod_{i=1}^{n}\left(z_{i}\right)^{\mu_{i}}, \quad z=\left(z_{1}, \ldots, z_{n}\right) \in \mathbb{T}^{n} .
$$

Then we see that $f \simeq p_{\mu}$, and hence there exists a function $\xi \in C_{\mathbb{R}}(X)$ such that $f=p_{\mu} \cdot \exp (i \xi)$. For each $z=\left(z_{1}, \ldots, z_{n}\right) \in \mathbb{T}^{n}$, we have

$$
\begin{aligned}
w(z) \cdot f\left(r_{\alpha}(z)\right) & =w(z) \cdot p_{\mu}\left(r_{\alpha}(z)\right) \cdot \exp \left(i \xi\left(r_{\alpha}(z)\right)\right) \\
& =w(z) \cdot p_{\mu}\left(\alpha_{1} z_{1}, \ldots, \alpha_{n} z_{n}\right) \cdot \exp \left(i \xi\left(r_{\alpha}(z)\right)\right) \\
& =w(z) \cdot \prod_{j=1}^{n}\left(\alpha_{j} z_{j}\right)^{\mu_{j}} \cdot \exp \left(i \xi\left(r_{\alpha}(z)\right)\right) \\
& =w(z) \cdot \prod_{j=1}^{n}\left(\alpha_{j}\right)^{\mu_{j}} \cdot \prod_{j=1}^{n}\left(z_{j}\right)^{\mu_{j}} \cdot \exp \left(i \xi\left(r_{\alpha}(z)\right)\right) \\
& =w(z) \cdot \prod_{j=1}^{n}\left(\alpha_{j}\right)^{\mu_{j}} \cdot p_{\mu}(z) \cdot \exp \left(i \xi\left(r_{\alpha}(z)\right)\right),
\end{aligned}
$$

which, by $(2.5)$, is equal to $\lambda \cdot f(z)=\lambda \cdot p_{\mu}(z) \cdot \exp (i \xi(z))$. It follows from this that

$$
w(z) \cdot \prod_{i=1}^{n}\left(\alpha_{i}\right)^{\mu_{i}} \cdot \exp \left(i \xi\left(r_{\alpha}(z)\right)\right)=\lambda \exp (i \xi(z)) .
$$


Hence, we see that the weight $w$ is written as

$$
w=\left(\lambda \prod_{i=1}^{n}\left(\alpha_{i}\right)^{-\mu_{i}}\right) \cdot \exp \left(-i\left(\xi \circ r_{\alpha}-\xi\right)\right)
$$

and thus it belongs to the right hand side of (2.4).

This completes the proof.

Theorem 2.8 and Theorem 2.9 reduce the description of $\operatorname{Ker} W_{T}$ to the detection of the real continuous coboundaries. The detection of coboundaries of a dynamical system is the subject of extensive study. We apply a few of those results to give a description of $\operatorname{Ker} W_{T}$. It should be pointed out that the same problem has been studied in [4] on composition operators on $L^{2}$ spaces induced by an invertible ergodic transformation $\tau$ on a probability space $(X, \mu)$. In [4], the weight function $w \in \operatorname{Map}(X, \mathbb{T})$, for which the composition operator $U_{\tau, w}: L^{2}(X, \mu) \rightarrow L^{2}(X, \mu)$ has an eigenfunction $g \in \operatorname{Map}(X, \mathbb{T})$, is called a projective coboundary of $\tau$.

Proposition 2.10. Let $T: X \rightarrow X$ be a continuous surjection on $a$ compact metric space $X$. Suppose that either of the following conditions (i) or (ii) holds:

(i) The map $T$ is minimal.

(ii) (a) There exists a probability measure $\nu$ with full support so that $T$ is $\nu$-ergodic, and

(b) The map $T$ is a local homeomorphism which is eventually onto.

Then, for a continuous function $f \in C_{\mathbb{R}}(X), f \in \mathrm{Cob}_{\mathbb{R}}(T)$ if and only if

$$
\sup _{n}\left\|\sum_{j=0}^{n} f \circ T^{j}\right\|_{\infty}<\infty .
$$

Proof. The conclusion under assumption (i) is a classical theorem due to Gottschalk-Hedlund [9], (see also [12, Theorem 2.9.4]). To derive the conclusion under assumption (ii), let $f \in C_{\mathbb{R}}(X)$ be a 
function such that

$$
\sup _{n}\left\|\sum_{j=0}^{n} f \circ T^{j}\right\|_{\infty}<\infty .
$$

By [6, Theorem 2], there exists a function $g \in L^{\infty}(X, \nu)$ such that $f=g \circ T-g, \nu$-almost everywhere. Since $T$ is a local homeomorphism which is eventually onto, we may apply [15, Theorem 6] to find a continuous function $G \in C_{\mathbb{R}}(X)$ such that $f=G \circ T-G$.

This completes the proof.

Our conclusions on positively expansive, open and eventually onto maps and minimal rotations on tori are summarized as follows.

\section{Corollary 2.11.}

(i) Let $T: X \rightarrow X$ be a positively expansive open surjection which is eventually onto such that the homomorphism $T^{*}-\mathrm{id}_{\check{\mathrm{H}}^{1}(X ; \mathbb{Z})}: \check{\mathrm{H}}^{1}(X ; \mathbb{Z}) \rightarrow \check{\mathrm{H}}^{1}(X ; \mathbb{Z})$ is injective. Then we have isomorphisms

$$
\operatorname{Im}\left(W_{T}\right) \cong \operatorname{Im}\left(T^{*}-\mathrm{id}\right)
$$

and

$$
\operatorname{Ker}\left(W_{T}\right) \cong \mathbb{T} \oplus \exp \left(i \operatorname{Cob}_{\mathbb{R}}(T)\right),
$$

where $\operatorname{Cob}_{\mathbb{R}}(X)=\left\{f \in C_{\mathbb{R}}(X) \mid \sup _{n}\left\|\sum_{j=0}^{n}\left(f \circ T^{j}\right)\right\|_{\infty}<\infty\right\}$.

(ii) Let $r_{\alpha}: \mathbb{T}^{n} \rightarrow \mathbb{T}^{n}$ be a minimal rotation on the $n$-dimensional torus $\mathbb{T}^{n}$ by an element $\alpha \in \mathbb{T}^{n}$. Then we have an isomorphism

$$
\mathcal{W}_{r_{\alpha}} \cong \mathbb{T} \oplus \exp \left(i \operatorname{Cob}_{\mathbb{R}}\left(r_{\alpha}\right)\right),
$$

where $\operatorname{Cob}_{\mathbb{R}}\left(r_{\alpha}\right)=\left\{f \in C_{\mathbb{R}}\left(\mathbb{T}^{n}\right) \mid \sup _{n}\left\|\sum_{j=0}^{n}\left(f \circ r_{\alpha}^{j}\right)\right\|_{\infty}<\infty\right\}$.

The referee and the author have conjectured that a positively expansive open surjection $T: X \rightarrow X$ of a compact metric space $X$ always induces the homomorphism $T^{*}: \check{\mathrm{H}}^{1}(X ; \mathbb{Z}) \rightarrow \check{\mathrm{H}}^{1}(X ; \mathbb{Z})$ with $T^{*}-\operatorname{id}_{\check{\mathrm{H}}^{1}(X ; \mathbb{Z})}$ being injective, yet the author has not been able to establish this. 
Acknowledgments. The author expresses his sincere gratitude to the referee for the valuable comments.

\section{REFERENCES}

1. N. Aoki, Expanding maps of solenoids, Monatsh. Math. 105 (1988), 1-34.

2. Topological dynamics, in Topics in general topology, K. Morita and J. Nagata, eds., North-Holland, Amsterdam, 1989.

3. N. Aoki and K. Hiraide, Topological theory of dynamical systems-Recent advances, North-Holland Math. Lib. 52 (1994), North-Holland.

4. L Baggett, On circle-valued cocycles of an ergodic measure-preserving transformation, Israel J. Math. 61 (1988), 29-38.

5. M. Bestvina, Characterizing $k$-dimensional universal Menger compactum, Mem. Amer. Math. Soc. 71, 1988.

6. F.E. Browder, On the iteration of transformations in non-compact minimal dynamical systems, Proc. Amer. Math. Soc. 9 (1958), 773-780.

7. A. Chigogidze, K. Kawamura and E.D. Tymchatyn, Menger manifolds, in Continua with Houston problem book, Marcel Dekker, New York, 1995.

8. E.M. Coven and W.L. Reddy, Positively expansive maps of compact manifolds, in Global Theory of Dynamical Systems, Lecture Notes in Math. 819 (1980), 96-110.

9. W.H. Gottschalk and G.A. Hedlund, Topological dynamics, Amer. Math. Soc. Colloq. Publ. 36, 1955.

10. K. Hiraide, Positively expansive open maps of Peano spaces, Topol. Appl. 37 (1990), 213-220.

11. K.H. Hoffmann and S. Morris, The structure of compact groups, Gruyter Stud. Math. 25, Walter de Gruyter, 1998.

12. A. Katok and B. Hasselblatt, An introduction to modern theory of dynamical systems, Cambridge University Press, Cambridge, 1995.

13. K. Kawamura, A survey on Menger manifold theory-Update, Topol. Appl. 101 (2000), 83-91.

14. P. Liardet and D. Volný, Sums of continuous and differentiable functions in dynamical systems, Israel J. Math. 98 (1997), 29-60.

15. A.N. Quas, Rigidity of continuous coboundaries, Bull. Lond. Math. Soc. 29 (1997), 595-600.

16. I. Rosenholtz, Local expansions, derivatives and fixed points, Fund. Math. 91 (1976), 1-4.

17. K. Sakai, Periodic points of positively expansive maps, Proc. Amer. Math. Soc. 94 (1985), 531-534.

18. Appl. 131 (2003), 15-31.

19. M. Shub, Endomorphisms of compact differentiable manifolds, Amer. J. Math. 91 (1969), 175-199. 
20. C.W. Stark, Minimal dynamics on Menger manifolds, Topol. Appl. 90 (1998), 21-30.

21. P. Walters, An introduction to ergodic theory, G.T.M. 79, Springer, Berlin, 1982.

Institute of Mathematics, University of Tsukuba, Tsukuba, Ibaraki 3058571, JAPAN

Email address: kawamura@math.tsukuba.ac.jp 